Pacific

Journal of

Mathematics

THE TANGENT GRUPOID OF A HEISENBERG MANIFOLD

RAPHAËL PONGE 


\title{
THE TANGENT GRUPOID OF A HEISENBERG MANIFOLD
}

\author{
RAPHAËL PONGE
}

\begin{abstract}
As a step towards proving an index theorem for hypoelliptic operators on Heisenberg manifolds, including for those on CR and contact manifolds, we construct an analogue for Heisenberg manifolds of Connes' tangent groupoid of a manifold. As is well known for a Heisenberg manifold $(M, H)$ the relevant notion of tangent bundle is rather that of a Lie group bundle of graded 2-step nilpotent Lie groups $G M$. We define the tangent groupoid of $(M, H)$ as a differentiable groupoid $\varphi_{H} M$ encoding the smooth deformation of $M \times M$ to $G M$. In particular, this construction makes a crucial use of a refined notion of privileged coordinates and of a tangent-approximation result for Heisenberg diffeomorphisms.
\end{abstract}

\section{Introduction}

A somewhat long standing open question is the existence of an index theorem for geometric operators on contact and CR manifolds. In this context the operators are not elliptic, so we cannot apply the classical index theorem of Atiyah-Singer [1968a; 1968b]. The natural pseudodifferential tool to deal with hypoelliptic operators on contact and CR manifolds is provided by the Heisenberg calculus of BealsGreiner [1988] and Taylor [1984]. The latter holds in full generality for Heisenberg manifolds, that is, manifolds $M$ together with a distinguished hyperplane bundle $H \subset T M$. This definition includes that of CR and contact manifolds, as well as that of codimension one foliations and confoliations. Therefore, what we would like to have is an analogue of the Atiyah-Singer theorem for hypoelliptic operators on Heisenberg manifolds.

There are various proofs of the Atiyah-Singer index theorem. A simple and fairly general proof is that of Connes [1994, Sect. II.5]. A salient feature in Connes' proof is the use of the tangent groupoid of a manifold, that is, the differentiable groupoid encoding the smooth deformation of $M \times M$ to $T M$ (see [Connes 1994; Hilsum and Skandalis 1987]).

In this paper, as a step towards proving an index theorem for hypoelliptic operators on Heisenberg manifolds, we construct an analogue for Heisenberg manifolds

MSC2000: primary 58H05; secondary 53C10, 53D10, $32 \mathrm{~V} 05$.

Keywords: differentiable groupoid, Heisenberg group, foliation, contact structure, CR structure. 
of Connes' tangent groupoid. The existence of such a tangent groupoid was conjectured by Bellaïche [1996, p. 74] for Carnot-Carathéodory manifolds and by Ponge [2000, p. 37] for Heisenberg manifolds. We also refer to Van Erp [2005] for an alternative description of this groupoid.

Our construction of the tangent groupoid of a Heisenberg manifold is carried out in two steps. The first step consists in giving a suitable description of the tangent Lie group bundle $G M$ of a Heisenberg manifold $(M, H)$. The latter is a bundle of graded 2-step nilpotent Lie groups and provides us with a more accurate tangent structure for Heisenberg manifolds than the classical tangent tangent space $T M$. There are various descriptions of GM in the literature (see, e.g., [Bellaïche 1996; Beals and Greiner 1988; Epstein et al. 1991; Folland and Stein 1974; Gromov 1996; Rockland 1987]).

Our description of GM stems from the existence of an intrinsic real-valued Levi form,

$$
\mathscr{L}: H \times H \longrightarrow T M / H .
$$

This is a 2-form on $H$ with values in the normal bundle $T M / H$ (see Lemma 2.3). It allows us to define the tangent Lie group bundle $G M$ as the bundle $(T M / H) \oplus H$ together with the grading and Lie group law, such that for sections $X_{0}, Y_{0}$ of $T M / H$ and sections $X^{\prime}, Y^{\prime}$ of $H$, we have

$$
\begin{gathered}
t .\left(X_{0}+X^{\prime}\right)=t^{2} X_{0}+t X^{\prime}, \quad t \in \mathbb{R}, \\
\left(X_{0}+X^{\prime}\right) \cdot\left(Y_{0}+Y^{\prime}\right)=X_{0}+Y_{0}+\frac{1}{2} \mathscr{L}\left(X^{\prime}, Y^{\prime}\right)+X^{\prime}+Y^{\prime} .
\end{gathered}
$$

This description of $G M$ is simple and is completely intrinsic. What is crucial, and more difficult, in the construction of the tangent groupoid is to relate the above description to the extrinsic tangent nilpotent approximations of some previous approaches (see, e.g., [Bellaïche 1996; Beals and Greiner 1988; Epstein et al. 1991; Folland and Stein 1974; Gromov 1996; Rockland 1987]). More precisely, given a point $x \in M$ the tangent Lie group $G_{x} M$ in these approaches is obtained as the Lie group associated to a Lie algebra of model vector fields in some privileged coordinates centered at $x$. We point out that by using a refined notion of privileged coordinates, which we call Heisenberg coordinates (see Definition 2.18), this approach coincides with ours (Proposition 2.20).

An important consequence of the equivalence between these two descriptions of $G M$ is a tangent approximation result for Heisenberg diffeomorphisms (Proposition 2.21). Namely, in Heisenberg coordinates a Heisenberg diffeomorphism is well approximated by a Lie group isomorphism between the tangent groups at the points. We really do need to use Heisenberg coordinates, because in general privileged coordinates we only get a Lie algebra isomorphism between the Lie algebras 
of the tangent group, and the corresponding Lie group isomorphism need not approximate the Heisenberg diffeomorphism (compare [Bellaïche 1996, Prop. 5.20]).

The second step is the construction of the tangent groupoid $\mathscr{G}_{H} M$ of a Heisenberg manifold $(M, H)$ as the $b$-differentiable groupoid $\mathscr{G}_{H} M$ that encodes the smooth deformation of $M \times M$ to $G M$ (Theorem 3.7). As an abstract groupoid the definition of $\varphi_{H} M$ is similar to that of Connes' tangent groupoid. In particular, at the set-theoretic level we have

$$
\mathscr{G}_{H} M=G M \sqcup(M \times M \times(0, \infty)) .
$$

In order to endow $\mathscr{G}_{H} M$ with a consistent topology, with a differentiable structure, as well as with a smooth composition map, we make crucial uses of the Heisenberg coordinates and of the tangent approximation of Heisenberg diffeomorphisms alluded to above. In this sense our construction differs from the usual construction of Connes' tangent groupoid. In addition, this construction is functorial with respect to Heisenberg diffeomorphisms (see Proposition 3.8 for the precise statement).

Beside potential applications towards an index theorem for hypoelliptic operators on Heisenberg manifolds, the construction of the tangent groupoid $\mathscr{G}_{H} M$ is also interesting from the point of view of Carnot-Carathéodory geometry. Namely, Gromov [1996] and Bellaïche [1996] proved that the tangent group at a point of a Carnot-Carathéodory manifold is tangent to the manifold in a topological sense (i.e. in terms of Gromov-Hausdorff limits). However, our tangent groupoid construction shows that, in the special case of Heisenberg manifolds, this tangency occurs in a differentiable sense.

More generally, it would be interesting to construct a tangent groupoid for more general Carnot-Carathéodory manifolds. As mentionned earlier this has been conjectured by Bellaiiche, but it is believed that the approach of this paper could be extended to deal with such a construction. Notice that in this setting the tangent Lie group bundle $G M$ should rather be an orbifold-bundle of Lie groups, but it should be an actual Lie group bundle when the Carathéodory distribution is equiregular in the sense of [Gromov 1996]. We hope to address these issues in a subsequent paper.

The remainder of the paper is organized as follows. In Section 2, after recalling the main facts about Heisenberg manifolds, we describe the tangent group bundle $G M$ of a Heisenberg manifold $(M, H)$ and prove our approximation result for Heisenberg diffeomorphisms. In Section 3 we construct the tangent groupoid of $(M, H)$ as a the differentiable groupoid that encodes the smooth deformation of $M \times M$ to $G M$.

Acknowledgements. I am grateful to Alain Connes, Pierre Julg, Henri Moscovici, Jean Renault and Erik Van Erp for discussions related to the subject matter of this paper. I also thank the hospitality of the IHÉS, where part of this paper was written. 


\section{The tangent Lie group bundle of a Heisenberg manifold}

In this section, after recalling the main definitions and examples of Heisenberg manifolds, we describe the tangent Lie group bundle of a Heisenberg manifold in terms of an intrinsic Levi form. We then relate this approach to the nilpotent approximation of vector fields from previous approaches by using Heisenberg coordinates, which refine the privileged coordinates of [Beals and Greiner 1988] and [Bellaïche 1996]. As a consequence we get a tangent-approximation result for Heisenberg diffeomorphism which will be crucial later in the construction of the tangent groupoid of a Heisenberg manifold.

\subsection{Heisenberg manifolds.}

Definition 2.1. (1) A Heisenberg manifold is a smooth manifold $M$ equipped with a distinguished hyperplane bundle $H \subset T M$.

(2) A Heisenberg diffeomorphism $\phi$ from a Heisenberg manifold $(M, H)$ onto another Heisenberg manifold $\left(M, H^{\prime}\right)$ is a diffeomorphism $\phi: M \rightarrow M^{\prime}$ such that $\phi^{*} H=H^{\prime}$.

Definition 2.2. Let $\left(M^{d+1}, H\right)$ be a Heisenberg manifold. Then:

(1) A (local) $H$-frame for $T M$ is a (local) frame $X_{0}, X_{1}, \ldots, X_{d}$ such that $X_{1}$, $\ldots, X_{d}$ span $H$.

(2) A local Heisenberg chart is a local chart with a local $H$-frame of $T M$ over its domain.

Following are the main examples of Heisenberg manifolds.

Heisenberg group. The $(2 n+1)$-dimensional Heisenberg group $\mathbb{W}^{2 n+1}$ consists in $\mathbb{R}^{2 n+1}=\mathbb{R} \times \mathbb{R}^{2 n}$ equipped with the group law,

$$
x \cdot y=\left(x_{0}+y_{0}+\sum_{1 \leq j \leq n}\left(x_{n+j} y_{j}-x_{j} y_{n+j}\right), x_{1}+y_{1}, \ldots, x_{2 n}+y_{2 n}\right) .
$$

A left-invariant basis for its Lie algebra $\mathfrak{h}^{2 n+1}$ is provided by the vector fields,

$$
X_{0}=\partial_{x_{0}}, \quad X_{j}=\partial_{x_{j}}+x_{n+j} \partial_{x_{0}}, \quad X_{n+j}=\partial_{x_{n+j}}-x_{j} \partial_{x_{0}},
$$

where $j$ ranges over $1, \ldots, n$. In particular, for $j, k=1, \ldots, n$ and $k \neq j$ we have the Heisenberg relations,

$$
\left[X_{j}, X_{n+k}\right]=-2 \delta_{j k} X_{0}, \quad\left[X_{0}, X_{j}\right]=\left[X_{j}, X_{k}\right]=\left[X_{n+j}, X_{n+k}\right]=0 .
$$

In particular, the subbundle spanned by the vector fields $X_{1}, \ldots, X_{2 n}$ gives rise to a left-invariant Heisenberg structure on $\mathbb{\boxplus}^{2 n+1}$. 
Foliations. A (smooth) foliation is a manifold $M$ together with a subbundle $\mathscr{F} \subset$ $T M$ which is integrable in the Frobenius sense, that is, the space of sections of $\mathscr{F}$ is closed under the Lie bracket of vector fields. Thus any codimension-1 foliation is a Heisenberg manifold.

Contact manifolds. Opposite to foliations are contact manifolds. A contact manifold is a Heisenberg manifold $\left(M^{2 n+1}, H\right)$ such that $H$ can be locally realized as the kernel of a contact form, that is, a 1-form $\theta$ such that $d \theta_{\mid H}$ is nondegenerate. When $M$ is orientable it is equivalent to require $H$ to be globally the kernel of a contact form. Furthermore, by Darboux's theorem any contact manifold is locally Heisenberg-diffeomorphic to the Heisenberg group $\mathbb{M}^{2 n+1}$ equipped with the standard contact form $\theta^{0}=d x_{0}+\sum_{j=1}^{n}\left(x_{j} d x_{n+j}-x_{n+j} d x_{j}\right)$.

Confoliations. According to Eliashberg and Thurston [1998], a confoliation on an oriented manifold $M^{2 n+1}$ is given by a global nonvanishing 1-form $\theta$ on $M$ such that $(d \theta)^{n} \wedge \theta \geq 0$. In particular, if we let $H=\operatorname{ker} \theta$ then $(M, H)$ is a Heisenberg manifold which turns to be a foliation when $d \theta \wedge \theta=0$ and a contact manifold when $(d \theta)^{n} \wedge \theta>0$.

CR manifolds. A CR structure on an orientable manifold $M^{2 n+1}$ is given by a rank- $n$ complex subbundle $T_{1,0} \subset T_{\mathbb{C}} M$ such that $T_{1,0}$ is integrable in Frobenius' sense and we have $T_{1,0} \cap T_{0,1}=\{0\}$, where we have let $T_{0,1}=\overline{T_{1,0}}$. Equivalently, the subbundle $H=\Re\left(T_{1,0} \oplus T_{0,1}\right)$ has the structure of a complex bundle of (real) dimension $2 n$. In particular, $(M, H)$ is a Heisenberg manifold.

The main example of a CR manifold is that of a (smooth) boundary $M=\partial D$ of a complex domain $D \subset \mathbb{C}^{n}$. In particular, when $D$ is strongly pseudoconvex (or strongly pseudoconcave) with defining function $\rho$ then $\theta=i(\partial-\bar{\partial}) \rho$ is a contact form on $M$.

2.2. The tangent Lie group bundle. The tangent Lie group bundle of a Heisenberg manifold $\left(M^{d+1}, H\right)$ can be described as follows.

First, we have:

Lemma 2.3. The Lie bracket on vector fields induces a TM/H-valued 2-form on $H$

$$
\mathscr{L}: H \times H \longrightarrow T M / H,
$$

such that, for any sections $X$ and $Y$ of $H$ near a point $m \in M$, we have

$$
\mathscr{L}_{m}(X(m), Y(m))=[X, Y](m) \bmod H_{m} .
$$

Proof. We only need to check that given two sections $X$ and $Y$ of $H$ near $m \in M$ the value of $[X, Y](m)$ modulo $H_{m}$ depends only on $X(m)$ and $Y(m)$. Indeed, if 
$f$ and $g$ are smooth functions near $m$ then we have

$$
\begin{aligned}
{[f X, g Y](m) } & =f(m) g(m)[X, Y](m)-Y(f)(m) X(m)+X(g)(m) Y(m) \\
& =f(m) g(m)[X, Y](m) \bmod H_{m} .
\end{aligned}
$$

This shows that if $X(\mathrm{~m})$ or $Y(\mathrm{~m})$ vanish then so does the class of $[X, Y](\mathrm{m})$ modulo $H_{m}$. Therefore, the latter only depends on the values of $X(m)$ and $Y(m)$. Hence the result.

Definition 2.4. The 2-form $\mathscr{L}$ is called the Levi form of $(M, H)$.

The Levi form $\mathscr{L}$ allows us to define a bundle $\mathfrak{g} M$ of graded Lie algebras by endowing $(T M / H) \oplus H$ with the smooth fields of Lie Brackets $[., .]_{\mathfrak{g} M}$ and gradings $X \rightarrow t . X, t \in \mathbb{R}$, such that, for $m \in M$ and $X_{0}, Y_{0}$ in $T_{m} M / H_{m}$ and $X^{\prime}, Y^{\prime}$ in $H_{m}$, we have

$$
\begin{gathered}
{\left[X_{0}+X^{\prime}, Y_{0}+Y^{\prime}\right]_{\mathfrak{g}_{m} M}=\mathscr{L}_{m}\left(X^{\prime}, Y^{\prime}\right),} \\
t .\left(X_{0}+X^{\prime}\right)=t^{2} X_{0}+t X^{\prime} .
\end{gathered}
$$

Definition 2.5. The bundle $\mathfrak{g} M$ is called the tangent Lie algebra bundle of $M$.

Proposition 2.6. The tangent Lie algebra bundle is 2-step nilpotent and contains the normal bundle $T M / H$ in its center.

Proof. It follows from 2.2 that $T M / H$ is contained in the center of $\mathfrak{g} M$ and that the Lie bracket $[., .]_{\mathfrak{g} M}$ maps to $T M / H$, so $\mathfrak{g} M$ is 2-step nilpotent.

Since $\mathfrak{g} M$ is nilpotent its associated graded Lie group bundle $G M$ can be described as follows. As a bundle $G M$ is $(T M / H) \oplus H$ and the exponential map is merely the identity. In particular the grading of $G M$ is as in 2.2. Moreover, as $\mathfrak{g} M$ is 2-step nilpotent the Campbell-Hausdorff formula gives

$$
(\exp X)(\exp Y)=\exp \left(X+Y+\frac{1}{2}[X, Y]\right) \quad \text { for sections } X, Y \text { of } \mathfrak{g} M .
$$

We thus deduce that the product on $G M$ is such that, for $m \in M$, and $X_{0}, Y_{0}$ in $T_{m} M / H_{m}$ and $X^{\prime}, Y^{\prime}$ in $H_{m}$, we have

$$
\left(X_{0}+X^{\prime}\right) \cdot\left(Y_{0}+X^{\prime}\right)=X_{0}+Y_{0}+\frac{1}{2} \mathscr{L}\left(X^{\prime}, Y^{\prime}\right)+X^{\prime}+Y^{\prime} .
$$

Definition 2.7. The bundle $G M$ is called the tangent Lie group bundle of $M$.

The fibers of $G M$ are classified by the Levi form $\mathscr{L}$ as follows:

Proposition 2.8. (1) The form $\mathscr{L}_{m}$ at $m \in M$ has rank $2 n$ if, and only if, $G_{m} M$ is isomorphic to $\mathbb{M}^{2 n+1} \times \mathbb{R}^{d-2 n}$ as a graded Lie group.

(2) The Levi form $\mathscr{L}$ has constant rank $2 n$ if, and only if, $G M$ is a fiber bundle with typical fiber $\mathbb{M}^{2 n+1} \times \mathbb{R}^{d-2 n}$. 
Proof. Let $g$ be a Riemannian metric on $H$. Notice that (1) is a pointwise statement and that (2) is a local statement, since $G M$ is a Lie group bundle already. Therefore, without any loss of generality, we may suppose that $T M / H$ is orientable, so that it admits a global nonvanishing section $X_{0}$. Then let $A$ denote the smooth section of End $H$ such that

$$
\mathscr{L}(X, Y)=g(X, A Y) X_{0} \quad \text { for sections } X, Y \text { of } H .
$$

Let $m \in M$. Since $\mathscr{L}_{m}$ is real-antisymmetric its rank has to be an even integer, say rk $\mathscr{L}_{m}=2 n$. Let us first assume that $\mathscr{L}_{m}$ is nondegenerate, i.e., $A_{m}$ is invertible. Let $A_{m}=J_{m}\left|A_{m}\right|$ be the polar decomposition of $A_{m}$ and on $H_{m}$ define the positive definite scalar product,

$$
h_{m}(X, Y)=\frac{1}{2} g_{m}\left(X,\left|A_{m}\right| Y\right) \quad X, Y \in H_{m} .
$$

Notice that $J_{m}$ is antisymmetric and unitary with respect to $h_{m}$, so we have $J_{m}^{2}=$ $-J_{m}^{t} J_{m}=-1$, that is, $J_{m}$ is a unitary complex structure on $H_{m}$. Therefore, we can construct a basis $X_{1}, \ldots, X_{2 n}$ of $H_{m}$ which is orthonormal with respect to $h_{m}$ and such that $X_{n+j}=J_{m} X_{j}$ for $j=1, \ldots, n$.

On the other hand, for $X$ and $Y$ in $H_{m} \subset \mathfrak{g}_{m} M$ we have

$$
[X, Y]_{\mathfrak{g}_{m} M}=\mathscr{L}_{m}(X, Y)=g_{m}\left(X, A_{m} Y\right) X_{0}=2 h_{m}\left(X, J_{m} Y\right) X_{0} .
$$

Thus, for $j=1, \ldots, n$ and $k=1, \ldots, n+j-1, n+j+1, \ldots, 2 n$ we get

$$
\begin{gathered}
{\left[X_{j}, X_{n+j}\right]_{\mathfrak{g}_{m} M}=2 h_{m}\left(X_{j}, J_{m}^{2} X_{j}\right) X_{0}=-2 h_{m}\left(X_{j}, X_{j}\right) X_{0}=-2 X_{0},} \\
{\left[X_{j}, X_{k}\right]_{\mathfrak{g}_{m} M}=h_{m}\left(X_{j}, J_{m} X_{k}\right) X_{0}=-h_{m}\left(X_{n+j}, X_{k}\right) X_{0}=0 .}
\end{gathered}
$$

These relations are the same as those in (2-1) for the Lie algebra of $\mathbb{H}^{2 n+1}$. Thus $G_{m} M$ is isomorphic to $\mathbb{W}^{2 n+1}$ as a graded Lie group.

Next, assume that $A_{m}$ has a nontrivial kernel. Then as $A_{m}$ is real antisymmetric with respect to $g_{m}$ we have an orthogonal direct sum $H_{m}=\operatorname{im} A_{m} \oplus \operatorname{ker} A_{m}$. In fact, it follows from (2-3) that if $X \in \operatorname{ker} A_{m}$ and $Y \in H_{m}$ then

$$
[X, Y]_{\mathfrak{g}_{m} M}=\mathscr{L}_{m}(X, Y)=g_{m}\left(X, A_{m} Y\right) X_{0}=0 .
$$

Thus ker $A_{m}$ is contained in the center of $\mathfrak{g}_{m} M$. Moreover, as $A_{m}$ is invertible on $\operatorname{im} A_{m}$ the same reasoning as above shows that the Lie subalgebra $\left(T_{m} M / H_{m}\right) \oplus$ $\operatorname{im} A_{m}$ is isomorphic to the (graded) Lie algebra $\mathfrak{h}^{2 n+1}$ of $\mathfrak{\boxplus}^{2 n+1}$. Therefore, $\mathfrak{g}_{m} M=$ $\left(T_{m} M / H_{m}\right) \oplus \operatorname{im} A_{m} \oplus \operatorname{ker} A_{m}$ is isomorphic to $\mathfrak{h}^{2 n+1} \times \mathbb{R}^{d-2 n}$, and so $G_{m} M$ is isomorphic to $\mathbb{U}^{2 n+1} \times \mathbb{R}^{d-2 n}$.

Conversely, suppose that $G_{m} M$ is isomorphic to $\mathfrak{h}^{2 n+1} \times \mathbb{R}^{d-2 n}$. Then $\mathfrak{g}_{m} M$ is isomorphic to $\mathfrak{h}^{2 n+1} \times \mathbb{R}^{d-2 n}$, so admits a basis $X_{0}, \ldots, X_{d}$ such that

$$
\left[X_{j}, X_{n+j}\right]_{\mathfrak{g}_{m} M}=-2 X_{0} \text { and }\left[X_{j}, X_{k}\right]_{\mathfrak{g}_{m} M}=\left[X_{l}, X_{k}\right]_{\mathfrak{g}_{m} M}=0,
$$


for $j=1, \ldots, n$ and $k=1, \ldots, d$ with $k \neq n+j$ and $l=2 n+1, \ldots, d$. Since $\mathscr{L}_{m}(X, Y)=[X, Y]_{\mathfrak{g}_{m} M}$ for $X$ and $Y$ in $H_{m}$ it follows from this that $\mathscr{L}_{m}$ has rank $2 n$. The proof of the first part of the proposition is thus complete.

Now, suppose that $\mathscr{L}$ has constant rank $2 n$. We have rk $A_{m}=2 n$ everywhere, so we get a vector bundle splitting $H=\operatorname{im} A \oplus \operatorname{ker} A$. Furthermore, the polar decomposition of $A_{m}$ is smooth with respect to $m$, i.e., $J$ and $|A|$ are smooth sections of End $H$. Therefore, the above process for constructing the basis $X_{0}, X_{1}, \ldots, X_{d}$ can be carried out near every point $m \in M$ in such way to yield a smooth $H$-frame satisfying the relations (2-6)-(2-7). Thus, near every point of $M$ we get a Lie group bundle trivialization of $G M$ as a trivial fiber bundle with fiber $\mathbb{H}^{2 n+1} \times \mathbb{R}^{d-2 n}$. Consequently, $G M$ is fiber bundle with typical fiber $\mathbb{H}^{2 n+1} \times \mathbb{R}^{d-2 n}$.

Conversely, assume that $G M$ is a fiber bundle with typical fiber $\mathbb{H}^{2 n+1} \times \mathbb{R}^{d-2 n}$. Then at every point $m \in M$ the Lie group $G_{m} M$ is isomorphic to $\mathbb{H}^{2 n+1} \times \mathbb{R}^{d-2 n}$, so it follows from the first part of the proposition that $\mathscr{L}$ has constant rank $2 n$.

In presence of a foliation or contact structure we have more precise results.

Proposition 2.9. Let $(M, H)$ be a Heisenberg manifold. Then the following are equivalent:

(1) $(M, H)$ is a foliation.

(2) $(M, H)$ is Levi flat, i.e., $\mathscr{L}$ vanishes.

(3) As a Lie group bundle GM agrees with $(T M / H) \oplus H$.

Proof. It follows from its definition that $\mathscr{L}$ vanishes if, and only if, for any sections $X$ and $Y$ of $H$ the Lie bracket vector field $[X, Y]$ is again a section of $H$, that is, if, and only if, $H$ defines a foliation.

On the other hand, in view of the definition of the group law of $G M$ the Levi form $\mathscr{L}$ vanishes if, and only if, the group law is $X . Y=X+Y$, that is, if, and only if, $G M$ is the Abelian Lie group bundle $(T M / H) \oplus H$.

Proposition 2.10. Suppose that $\left(M^{2 n+1}, H\right)$ is a Heisenberg manifold. Then the following are equivalent:

(1) $(M, H)$ is a contact manifold.

(2) The Levi form $\mathscr{L}$ is (everywhere) nondegenerate.

(3) The Lie group tangent bundle GM is a fiber bundle with typical fiber $\mathbb{H}^{2 n+1}$.

Proof. Since the equivalence of (2) and (3) follows from Proposition 2.8, we only have to prove that (1) and (2) are equivalent. Since these are local statements we may assume that $T M / H$ is orientable, i.e., there exists a global nonzero 1-form $\theta$ such that $H=\operatorname{ker} \theta$. As any nonzero 1 -form annihiliting $H$ is a nonzero multiple of $\theta$ we see that $(M, H)$ is a contact manifold if, and only if, $\theta$ is a contact form. 
Now, for any sections $X$ and $Y$ of $H$ we have

$$
\mathscr{L}(X, Y)=\theta([X, Y]) X_{0}=-d \theta(X, Y) X_{0} .
$$

This shows that $\mathscr{L}$ and $d \theta_{\left.\right|_{H}}$ have same rank, so $\theta$ is a contact form if, and only if, $\mathscr{L}$ is everywhere nondegenerate. This proves the equivalence of (1) and (2) and thus completes the proof of the proposition.

Finally, let $\phi:(M, H) \rightarrow\left(M^{\prime}, H^{\prime}\right)$ be a Heisenberg diffeomorphism from $(M, H)$ onto another Heisenberg manifold $\left(M^{\prime}, H^{\prime}\right)$. Since we have $\phi_{*} H=H^{\prime}$, we see that $\phi^{\prime}$ induces a smooth vector bundle isomorphism $\bar{\phi}$ from $T M / H$ onto $T M^{\prime} / H^{\prime}$.

Definition 2.11. We let $\phi_{H}^{\prime}:(T M / H) \oplus H \rightarrow\left(T M^{\prime} / H^{\prime}\right) \oplus H^{\prime}$ denote the vector bundle isomorphism such that

$$
\phi_{H}^{\prime}(m)\left(X_{0}+X^{\prime}\right)=\bar{\phi}^{\prime}(m) X_{0}+\phi^{\prime}(m) X^{\prime}
$$

for any $m \in M, X_{0} \in T_{m} / H_{m}$, and $X^{\prime} \in H_{m}$.

Proposition 2.12. The vector bundle isomorphism $\phi_{H}^{\prime}$ is an isomorphism of graded Lie group bundles from $G M$ onto $G M^{\prime}$.

Proof. If $X$ and $Y$ are sections of $H$ then we have

(2-12) $\mathscr{L}\left(\phi_{H}^{\prime}(X), \phi_{H}^{\prime}(Y)\right)=\left[\phi_{*} X, \phi_{*} Y\right]=\phi_{*}^{\prime}[X, Y]=\phi_{H}^{\prime}\left(\mathscr{L}_{m}(X, Y)\right) \bmod H^{\prime}$.

In view of (2-2) this implies that $\phi_{H}^{\prime}$ is a Lie group bundle isomorphism from $G M$ onto $G M^{\prime}$. Furthemore, it follows from (2-11) that, for any $t \in \mathbb{R}$ and any section $X$ of $G M$, we have $\phi_{H}^{\prime}(t . X)=t . \phi_{H}^{\prime}(X)$, i.e., $\phi_{H}^{\prime}$ is graded.

Corollary 2.13. The Lie group bundle isomorphism class of GM depends only on the Heisenberg diffeomorphism class of $(M, H)$.

2.3. Heisenberg coordinates and nilpotent approximations of vector fields. In the sequel it will be useful to combine the above intrinsic description of $G M$ with a more extrinsic description of the tangent Lie group at a point in terms of the Lie group associated to a nilpotent Lie algebra of model vector field. Incidentally, this will show that our approach is equivalent to previous ones [Beals and Greiner 1988; Bellaïche 1996; Epstein et al. 1991; Folland and Stein 1974; Gromov 1996; Rockland 1987].

First, pick $m \in M$ and let us describe $\mathfrak{g}_{m} M$ as the graded Lie algebra of leftinvariant vector field on $G_{m} M$ by identifying any $X \in \mathfrak{g}_{m} M$ with the left-invariant vector field $L_{X}$ on $G_{m} M$ given by

$$
L_{X} f(x)=\left.\frac{d}{d t} f(t \exp (X) \cdot x)\right|_{t=0}=\left.\frac{d}{d t} f(t X \cdot x)\right|_{t=0}, \quad f \in C^{\infty}\left(G_{m} M\right) .
$$


This allows us to associate, to any vector field $X$ near $m$, a unique left-invariant vector field $X^{m}$ on $G_{m} M$ such that

$$
X^{m}= \begin{cases}L_{X_{0}(m)} & \text { if } X(m) \notin H_{m} \\ L_{X(m)} & \text { otherwise }\end{cases}
$$

where $X_{0}(m)$ denotes the class of $X(m)$ modulo $H_{m}$.

Definition 2.14. The left-invariant vector field $X^{m}$ is called the model vector field of $X$ at $m$.

Let us look at this construction in terms of an $H$-frame $X_{0}, \ldots, X_{d}$ near $m$, i.e., of a local trivialization of the vector bundle $(T M / H) \oplus H$. For $j, k=1, \ldots, d$ set

$$
\mathscr{L}\left(X_{j}, X_{k}\right)=\left[X_{j}, X_{k}\right]=L_{j k} X_{0} \bmod H .
$$

With respect to the coordinate system $\left(x_{0}, \ldots, x_{d}\right)$ corresponding to $X_{0}(m), \ldots$, $X_{d}(m)$ we can write the product law of $G_{m} M$ as

$$
x \cdot y=\left(x_{0}+\frac{1}{2} \sum_{j, k=1}^{d} L_{j k} x_{j} y_{k}, x_{1}+y_{1}, \ldots, x_{d}+y_{d}\right) .
$$

The vector fields $X_{j}^{m}, j=1, \ldots, d$, in (2-13) are just the left-invariant vector fields corresponding to the vectors of the canonical basis $e_{1}, \ldots, e_{d}$, i.e., we have

$$
X_{0}^{m}=\partial_{x_{0}} \quad \text { and } \quad X_{j}^{m}=\partial_{x_{j}}-\left(\frac{1}{2} \sum_{k=1}^{d} L_{j k} x_{k}\right) \partial_{x_{0}}, \quad 1 \leq j \leq d .
$$

In particular, for $j, k=1, \ldots, d$, we have the relations

$$
\left[X_{j}^{m}, X_{k}^{m}\right]=L_{j k}(m) X_{0}^{m} \quad \text { and } \quad\left[X_{j}^{m}, X_{0}^{m}\right]=0 .
$$

Let $X$ be a vector field near $m$. Then $X$ is of the form $X=a_{0}(x) X_{0}+\cdots+$ $a_{d}(x) X_{d}$ near $m$, and its model vector field $X^{m}$ is thus given by the formula

$$
X^{m}= \begin{cases}a_{0}(m) X_{0}^{m} & \text { if } a_{0}(m) \neq 0, \\ a_{1}(m) X_{1}^{m}+\cdots+a_{d}(m) X_{d}^{m} & \text { otherwise. }\end{cases}
$$

Now, let $\kappa: \operatorname{dom} \kappa \rightarrow U$ be a Heisenberg chart near $m=\kappa^{-1}(u)$ and let $X_{0}, \ldots, X_{d}$ be the associated $H$-frame of $T U$. There exists a unique affine coordinate change $v \rightarrow \psi_{u}(v)$ such that $\psi_{u}(u)=0$ and $\psi_{u *} X_{j}(0)=\partial_{x_{j}}$ for $j=0,1, \ldots, d$. Indeed, if for $j=1, \ldots, d$ we set $X_{j}(x)=\sum_{k=0}^{d} B_{j k}(x) \partial_{x_{k}}$ then we have

$$
\psi_{u}(x)=A(u)(x-u), \quad \text { where } A(u)=\left(B(u)^{t}\right)^{-1} .
$$


Definition 2.15 [Beals and Greiner 1988].

(1) The coordinates provided by $\psi_{u}$ are called the privileged coordinates at $u$ with respect to the $H$-frame $X_{0}, \ldots, X_{d}$.

(2) The map $\psi_{u}$ is called the privileged coordinate map at $u$ with respect to the $H$-frame $X_{0}, \ldots, X_{d}$.

Remark 2.16. In [Beals and Greiner 1988] the privileged coordinates at $u$ are called $u$-coordinates, but in the special case of a Heisenberg manifold they correspond to the privileged coordinates of [Bellaïche 1996] and [Gromov 1996].

Notice that in the privileged coordinates at $u$ we can write

$$
X_{j}=\partial_{x_{j}}+\sum_{k=0}^{d} a_{j k}(x) \partial_{x_{k}}, \quad j=0,1, \ldots d,
$$

where the $a_{j k}$ 's are smooth functions such that $a_{j k}(0)=0$.

Next, on $\mathbb{R}^{d+1}$ we consider the dilations

$$
\delta_{t}(x)=t \cdot x=\left(t^{2} x_{0}, t x_{1}, \ldots, t x_{d}\right), \quad t \in \mathbb{R},
$$

with respect to which $\partial_{x_{0}}$ is homogeneous of degree -2 , while $\partial_{x_{1}}, \ldots, \partial_{x_{d}}$ are homogeneous of degree -1 . Therefore, we may let

$$
\begin{aligned}
& X_{0}^{(u)}=\lim _{t \rightarrow 0} t^{2} \delta_{t}^{*} X_{0}=\partial_{x_{0}}, \\
& X_{j}^{(u)}=\lim _{t \rightarrow 0} t \delta_{t}^{*} X_{j}=\partial_{x_{j}}+\sum_{k=1}^{d} b_{j k} x_{k} \partial_{x_{0}}, \quad j=1, \ldots, d,
\end{aligned}
$$

where $b_{j k}=\partial_{x_{k}} a_{j 0}(0)$ for $j, k=1, \ldots, d$. In fact, for any vector field $X=$ $a_{0}(x) X_{0}+\cdots+a_{d}(x) X_{d}$ we have

$$
\begin{aligned}
& \lim _{t \rightarrow 0} t^{2} \delta_{t}^{*} X=a_{0}(0) X_{0}^{(u)}, \\
& \lim _{t \rightarrow 0} t^{-1} \delta_{t}^{*} X=a_{1}(0) X_{1}^{(u)}+\cdots+a_{d}(0) X_{d}^{(u)} \quad \text { when } a_{0}(0)=0 .
\end{aligned}
$$

Observe that $X_{0}^{(u)}$ is homogeneous of degree -2 and $X_{1}^{(u)}, \ldots, X_{d}^{(u)}$ are homogeneous of degree -1 . Moreover, for $j, k=1, \ldots, d$, we have

$$
\left[X_{j}^{(u)}, X_{0}^{(u)}\right]=0 \quad \text { and } \quad\left[X_{j}^{(u)}, X_{0}^{(u)}\right]=\left(b_{k j}-b_{j k}\right) X_{0}^{(u)} .
$$

Thus, the linear space spanned by $X_{0}^{(u)}, X_{1}^{(u)}, \ldots, X_{d}^{(u)}$ is a graded 2-step nilpotent Lie algebra $\mathfrak{g}^{(u)}$. In particular, $\mathfrak{g}^{(u)}$ is the Lie algebra of left-invariant vector fields over the graded Lie group $G^{(u)}$, consisting of $\mathbb{R}^{d+1}$ equipped with the grading (2-17) and the group law

$$
x \cdot y=\left(x_{0}+\sum_{j, k=1}^{d} b_{k j} x_{j} y_{k}, x_{1}+y_{1}, \ldots, x_{d}+y_{d}\right) .
$$


Now, if near $m$ we set $\mathscr{L}\left(X_{j}, X_{k}\right)=\left[X_{j}, X_{k}\right]=L_{j k} X_{0} \bmod H$, then we have

$$
\left[X_{j}^{(u)}, X_{k}^{(u)}\right]=\lim _{t \rightarrow 0}\left[t \delta_{t}^{*} X_{j}, t \delta_{t}^{*} X_{k}\right]=\lim _{t \rightarrow 0} t^{2} \delta_{t}^{*}\left(L_{j k} X_{0}\right)=L_{j k}(m) X_{0}^{(u)} .
$$

Comparing this with (2-15) and (2-21) shows that $\mathfrak{g}^{(u)}$ has the same constants of structure as $\mathfrak{g}_{m} M$, and is therefore isomorphic to it. Consequently, the Lie groups $G^{(u)}$ and $G_{m} M$ are isomorphic. An explicit isomorphism can be obtained as follows.

Lemma 2.17. Consider a diffeomorphism $\phi: \mathbb{R}^{d+1} \rightarrow \mathbb{R}^{d+1}$ of the form

$$
\phi\left(x_{0}, \ldots, x_{d}\right)=\left(x_{0}+\frac{1}{2} c_{j k} x_{j} x_{k}, x_{1}, \ldots, x_{d}\right),
$$

where $c=\left(c_{j k}\right), c^{t}=c$, is a symmetric matrix in $M_{d}(\mathbb{R})$. Then $\phi$ is a graded isomorphism from $G^{(u)}$ onto the Lie group $G$ consisting of $\mathbb{R}^{d+1}$ equipped with the group law,

$$
x . y=\left(x_{0}+y_{0}+\sum_{j, k=1}^{d}\left(b_{k j}+c_{k j}\right) x_{j} y_{k}, x_{1}+y_{1}, \ldots, x_{d}+y_{d}\right) .
$$

Moreover, under $\phi$ the vector fields $X_{0}^{(u)}, \ldots, X_{d}^{(u)}$ transform into

$$
\begin{gathered}
\phi_{*} X_{0}^{(u)}=\frac{\partial}{\partial x_{0}}, \\
\phi_{*} X_{j}^{(u)}=\partial_{x_{j}}+\sum_{k=1}^{d}\left(b_{j k}+c_{j k}\right) x_{k} \partial_{x_{0}}, \quad j=1, \ldots, d .
\end{gathered}
$$

Proof. First, since $\phi(t \cdot x)=t \cdot \phi(x)$ for any $t \in \mathbb{R}$, we see that $\phi$ is graded. Second, for $x$ and $y$ in $\mathbb{R}^{d+1}$ the product $\phi(x) \cdot \phi(y)$ is equal to

$$
\begin{aligned}
& \phi\left(x_{0}+y_{0}+\sum_{j, k=1}^{d} b_{k j} x_{j} y_{k}, x_{1}+y_{1}, \ldots, x_{d}+y_{d}\right) \\
& =\left(x_{0}+y_{0}+\sum_{j, k=1}^{d} b_{k j} x_{j} y_{k}+\frac{1}{2} \sum_{j, k=1}^{d} c_{j k}\left(x_{j}+y_{j}\right)\left(x_{k}+y_{k}\right), x_{1}+y_{1}, \ldots, x_{d}+y_{d}\right) \\
& =\left(x_{0}+\frac{1}{2} \sum_{j, k=1}^{d} c_{j k} x_{j} x_{k}+y_{0}+\frac{1}{2} \sum_{j, k=1}^{d}\left(c_{j k} y_{j} y_{k}+\left(b_{k j}+c_{k j}\right) x_{j} y_{k}\right), x_{1}+y_{1}, \ldots, x_{d}+y_{d}\right) .
\end{aligned}
$$

Thus, in view of the law group of $G$ we have $\phi(x \cdot y)=\phi(x) \cdot \phi(y)$ and $\phi$ is a Lie group isomorphism. Consequently, for each $j=0, \ldots, d$, the vector field

$$
\phi_{*} X_{j}^{(u)}=\phi^{\prime}\left(\phi^{-1}(x)\right)\left(X_{j}\left(\phi^{-1}(x)\right)\right)
$$

is left-invariant on $G$. In fact, as $\phi^{\prime}(0)=\mathrm{id}$ and $X_{j}^{(u)}(0)=\partial_{x_{j}}$ we see that $\phi_{*} X_{j}^{(u)}$ is the left-invariant vector field on $G$ that coincides with $\partial_{x_{j}}$ at $x=0$. Therefore, by 
substituting $b_{j k}+c_{j k}$ for $b_{j k}$ in (2-18)-(2-19), we get the formulas (2-25)-(2-26) for $\phi_{*} X_{j}^{(u)}$. The lemma is thus proved.

Since by (2-21) and (2-22) we have $L_{j k}=b_{k j}-b_{j k}$ for $j, k=1, \ldots, d$, we deduce from Lemma 2.17 that an isomorphism of graded Lie groups from $G^{(u)}$ onto $G_{m} M$ is given by

$$
\phi_{u}\left(x_{0}, \ldots, x_{d}\right)=\left(x_{0}-\frac{1}{4} \sum_{j, k=1}^{d}\left(b_{j k}+b_{k j}\right) x_{j} x_{k}, x_{1}, \ldots, x_{d}\right) .
$$

Definition 2.18. Let $\varepsilon_{u}=\phi_{u} \circ \psi_{u}$.

(1) The new coordinates provided by $\varepsilon_{u}$ are called Heisenberg coordinates at $u$ with respect to the $H$-frame $X_{0}, \ldots, X_{d}$.

(2) The map $\varepsilon_{u}$ is called the $u$-Heisenberg coordinate map.

Remark 2.19. The Heisenberg coordinates were first introduced in [Beals and Greiner 1988], where they were called "antisymmetric $u$-coordinates" and used as a technical tool for inverting the principal symbol of a hypoelliptic sublaplacian.

Next, Lemma 2.17 also tells us that

$$
\begin{aligned}
& \phi_{*} X_{0}^{(u)}=\partial_{x_{0}}=X_{0}^{m}, \\
& \phi_{*} X_{j}^{(u)}=\partial_{x_{j}}-\frac{1}{2} \sum_{k=1}^{d} L_{j k} x_{k} \partial_{x_{0}}=X_{j}^{m}, \quad j=1, \ldots, d .
\end{aligned}
$$

Since $\phi_{u}$ commutes with the Heisenberg dilations (2-17), by using (2-18) and (2-19) we get

$$
\lim _{t \rightarrow 0} t^{2} \delta_{t}^{*} \phi_{u *} X_{0}^{(u)}=X_{0}^{m} \quad \text { and } \quad \lim _{t \rightarrow 0} t \delta_{t}^{*} \phi_{u *} X_{j}^{(u)}=X_{j}^{m}, \quad j=1, \ldots, d .
$$

Combining this with (2-16) and (2-20) shows that, for any vector field $X$ near $m$, in Heisenberg coordinates at $m$ we have, as $t \rightarrow 0$,

$$
\delta_{t}^{*} X= \begin{cases}t^{-2} X^{m}+O\left(t^{-1}\right) & \text { if } X(m) \in H_{m} \\ t^{-1} X^{m}+O(1) & \text { otherwise }\end{cases}
$$

Therefore, we obtain:

Proposition 2.20. In Heisenberg coordinates centered at $m=\kappa^{-1}(u)$, the tangent Lie group $G_{m} M$ coincides with $G^{(u)}$.

2.4. Tangent approximation of Heisenberg diffeomorphisms. If $\phi: M \rightarrow M^{\prime}$ is a smooth map between (standard) smooth manifolds, then for any $m \in M$ the derivative $\phi^{\prime}(m)$ yields a tangent linear approximation for $\phi$ in local coordinates 
around $m$. We shall now prove an analogous result in the Heisenberg setting. To this end, it will be useful to endow $\mathbb{R}^{d+1}$ with the pseudonorm,

$$
\|x\|=\left(x_{0}^{2}+\left(x_{1}^{2}+\ldots+x_{d}^{2}\right)^{2}\right)^{1 / 4}, \quad x \in \mathbb{R}^{d+1},
$$

so that, for any $x \in \mathbb{R}^{d+1}$ and any $t \in \mathbb{R}$, we have

$$
\|t \cdot x\|=|t|\|x\| .
$$

From now on we let $\phi:(M, H) \rightarrow\left(M^{\prime}, H^{\prime}\right)$ be a Heisenberg diffeomorphism from $(M, H)$ to another Heisenberg manifold $\left(M^{\prime}, H^{\prime}\right)$.

Proposition 2.21. Let $m \in M$ and set $m^{\prime}=\phi(m)$. Then, in Heisenberg coordinates at $m$ and at $m^{\prime}$, the diffeomorphism $\phi(x)$ has a behavior near $x=0$ of the form

$$
\phi(x)=\phi_{H}^{\prime}(0) x+\left(O\left(\|x\|^{3}\right), O\left(\|x\|^{2}\right), \ldots, O\left(\|x\|^{2}\right)\right),
$$

where $\phi_{H}$ is as in Definition 2.11. In particular, there is no term of the form $x_{j} x_{k}$, $1 \leq j, k \leq d$, in the Taylor expansion of $\phi_{0}(x)$ at $x=0$.

Proof. Let $X_{0}, \ldots, X_{d}$ be an $H$-frame of $T M$ over a Heisenberg chart $\kappa$ near $m$ and let $Y_{0}, \ldots, Y_{d}$ be an $H^{\prime}$-frame of $T M^{\prime}$ over a Heisenberg chart $\kappa_{1}$ near $m^{\prime}$. Set $u=\kappa(m)$, so that in privileged coordinates at $u$ we have $X_{j}(0)=\partial_{x_{j}}$ for $j=0, \ldots, d$. As the change of variables $\phi_{u}$ from privileged coordinates to Heisenberg coordinates at $u$ is such that $\phi_{u}(0)=0$ and $\phi_{u}^{\prime}(0)=\mathrm{id}$, we see that in Heisenberg coordinates at $m$ we also have $X_{j}(0)=\partial_{x_{j}}$ for $j=0, \ldots, d$. Similarly, in Heisenberg coordinates at $m^{\prime}$ we have $Y_{j}(0)=\partial_{x_{j}}$ for $j=0, \ldots, d$. As $\phi^{\prime}(0)$ maps $H_{0}$ to $H_{0}^{\prime}$ it then follows that, with respect to the basis $\partial_{x_{0}}, \ldots, \partial_{x_{d}}$, the matrices of $\phi^{\prime}(0)$ and $\phi_{H}^{\prime}(0)$ take the forms

$$
\phi^{\prime}(0)=\left(\begin{array}{cc}
a_{00} & 0 \\
b & A_{\|}
\end{array}\right) \quad \text { and } \quad \phi_{H}^{\prime}(0)=\left(\begin{array}{cc}
a_{00} & 0 \\
0 & A_{\|}
\end{array}\right),
$$

for some scalar $a_{00} \neq 0$ and some matrices $b \in M_{d 1}(\mathbb{R})$ and $A_{\|} \in G L_{d}(\mathbb{R})$. In particular, we have $\phi^{\prime}(0) x=\phi_{H}^{\prime}(0) x+x_{0}\left(0, b_{1}, \ldots, b_{d}\right)$. Thus, the Taylor expansion of $\phi(x)$ at $x=0$ takes the form

$$
\phi(x)=\hat{\phi}(x)+\theta(x), \quad \hat{\phi}(x)=\left(x_{0}+\frac{1}{2} \sum_{j, k=1}^{d} c_{j k} x_{j} x_{k}, x_{1}, \ldots, x_{d}\right),
$$

where $c_{j k}=\partial_{x_{j}, x_{k}}^{2} \phi_{0}(0)$ and $\theta(x)=\left(\theta_{0}(x), \ldots, \theta_{d}(x)\right)$ is such that

$$
\begin{aligned}
& \theta_{0}(x)=O\left(\left|x_{0}\right||x|+|x|^{3}\right)=O\left(\|x\|^{3}\right), \\
& \theta_{j}(x)=O\left(\left|x_{0}\right|+|x|^{2}\right)=O\left(\|x\|^{2}\right), \quad j=1, \ldots, d .
\end{aligned}
$$

To complete the proof we need only to show that $c_{j k}=0$ for $j, k=1, \ldots, d$. Possibly after replacing $\phi$ by $\phi_{H}^{\prime}(0)^{-1} \circ \phi$ we may assume that $\phi_{H}^{\prime}(0)=$ id. Since 
by Proposition $2.12 \phi_{H}^{\prime}(0)$ is a Lie group isomorphism from $G=G_{0} M$ onto $G^{\prime}=$ $G_{0} M^{\prime}$, this implies that $G$ and $G^{\prime}$ have the same group law, namely,

$$
x \cdot y=\left(x_{0}+y_{0}+\frac{1}{2} \sum_{j, k=1}^{d} L_{j k} x_{j} x_{k}, x_{1}+y_{1}, \ldots, x_{d}+y_{d}\right),
$$

where the structure constants $L_{j k}$ are such that

$$
\mathscr{L}\left(X_{j}, X_{k}\right)(0)=\mathscr{L}\left(Y_{j}, Y_{k}\right)(0)=L_{j k} X_{0}(0) .
$$

Therefore, using (2-14) we see that, at the level of the model vector fields (2-13), we have

$$
\begin{aligned}
& X_{0}^{m}=Y_{0}^{m^{\prime}}=\partial_{x_{0}}, \\
& X_{j}^{m}=Y_{j}^{m^{\prime}}=\partial_{x_{j}}-\frac{1}{2} \sum_{k=1}^{d} L_{j k} x_{k} \partial_{x_{0}}, \quad j=1, \ldots, d .
\end{aligned}
$$

As in (2-31) $\phi_{H}^{\prime}(0)$ is the diagonal part of $\phi^{\prime}(0)$ we have $\phi_{*} X_{0}(0)=Y_{j}(0) \bmod H_{0}^{\prime}$ and $\phi_{*} X_{0}(0)=Y_{j}(0)$ for $j=1, \ldots, d$. Therefore, using (2-13) we obtain

$$
\left(\phi_{*} X_{j}\right)^{m^{\prime}}=Y_{j}^{m^{\prime}}=X_{j}^{m} \quad \text { for } j=0, \ldots, d .
$$

On the other hand, as we are using Heisenberg coordinates both at $m$ and $m^{\prime}$, from (2-28) we get

$$
X_{j}^{m}=\lim _{t \rightarrow 0} t \delta_{t}^{*} X_{j} \quad \text { and } \quad\left(\phi_{*} X_{j}\right)^{m^{\prime}}=\lim _{t \rightarrow 0} t \delta_{t}^{*} \phi_{*} X_{j}=\lim _{t \rightarrow 0}\left(\delta_{t}^{-1} \circ \phi \circ \delta_{t}\right)_{*}\left(t \delta_{t}^{*} X_{j}\right) .
$$

Since (2-32)-(2-34) imply that $\lim _{t \rightarrow 0} \delta_{t}^{-1} \circ \phi \circ \delta_{t}=\hat{\phi}$, we see that

$$
\left(\phi_{*} X_{j}\right)^{m^{\prime}}=\lim _{t \rightarrow 0}\left(\delta_{t}^{-1} \circ \phi \circ \delta_{t}\right)_{*} \lim _{t \rightarrow 0}\left(t \delta_{t}^{*} X_{j}\right)=\hat{\phi}_{*} X_{j}^{m} .
$$

Combining this with (2-36) we then get

$$
\hat{\phi}_{*} X_{j}^{m}=\left(\phi_{*} X_{j}\right)^{m^{\prime}}=X_{j}^{m} \quad \text { for } j=1, \ldots, d .
$$

Now, the form of $\hat{\phi}$ in (2-32) allows us to apply Lemma 2.17 to get

$$
\hat{\phi}_{*} X_{j}^{m}=\partial_{x_{j}}+\sum_{k=1}^{d}\left(-\frac{1}{2} L_{j k}+c_{j k}\right) x_{k} \partial_{x_{0}} .
$$

Combining with (2-35) and (2-37) then gives $L_{j k}=L_{j k}-2 c_{j k}$, from which we deduce that $c_{j k}=0$ for $j, k=1, \ldots, d$. The proof is thus complete.

Remark 2.22. An asymptotics similar to (2-30) is given in [Bellaïche 1996, Proposition 5.20] by using privileged coordinates at $u$ and $u^{\prime}=\kappa_{1}\left(m^{\prime}\right)$, but the leading term there is only a Lie algebra isomorphism from $\mathfrak{g}^{(u)}$ onto $\mathfrak{g}^{\left(u^{\prime}\right)}$. It is only in Heisenberg coordinates that we recover the Lie group isomorphism $\phi_{H}^{\prime}(m)$ as the leading term of the asymptotics.

Finally, for future use we mention the following version of Proposition 2.21. 
Proposition 2.23. In local coordinates and as $t \rightarrow 0$ we have

$$
t^{-1} \cdot \varepsilon_{\phi(u)} \circ \phi \circ \varepsilon_{u}^{-1}(t \cdot x)=\left(\varepsilon_{\phi(u)} \circ \phi \circ \varepsilon_{u}^{-1}\right)_{H}^{\prime}(0) x+O(t),
$$

locally uniformly with respect to $u$ and $x$.

Proof. By combining Proposition 2.21 and (2-29) we get

$$
t^{-1} \cdot \varepsilon_{\phi(u)} \circ \phi \circ \varepsilon_{u}^{-1}(t \cdot x)=\left(\varepsilon_{\phi(u)} \circ \phi \circ \varepsilon_{u}^{-1}\right)_{H}^{\prime}(0) x+O(t) .
$$

A priori this holds only pointwise with respect to $u$ and $x$. However, the asymptotic bound above comes from remainder terms in Taylor formulas at $t=0$ for components of $\Psi(u, x, t):=\varepsilon_{\phi(u)} \circ \phi \circ \varepsilon_{u}^{-1}(t \cdot x)$. Since $\Psi$ is smooth with respect to $u$ and $x$, it follows that the bounds in (2-38) are locally uniform with respect to $u$ and $x$. Hence the result.

\section{The tangent groupoid of a Heisenberg manifold}

In this section we construct the tangent groupoid of a Heisenberg manifold $(M, H)$ as a groupoid encoding the smooth deformation of $M \times M$ to $G M$. In this construction a crucial use is made of Heisenberg coordinates and of the tangent approximation of Heisenberg diffeomorphisms provided by Proposition 2.21.

3.1. Differentiable groupoids. Here we recall the main definitions on groupoids and illustrate them with the example of Connes' tangent groupoid.

Definition 3.1. A groupoid consists of a set $\mathscr{G}$, a distinguished subset $\mathscr{G}^{(0)} \subset \mathscr{G}$, two maps $r$ and $s$ from $\mathscr{G}$ to $\mathscr{G}^{(0)}$ (called the range and source maps) and a composition map,

$$
\circ: \mathscr{G}^{(2)}=\left\{\left(\gamma_{1}, \gamma_{2}\right) \in \mathscr{G} \times \mathscr{G} \mid s\left(\gamma_{1}\right)=r\left(\gamma_{2}\right)\right\} \longrightarrow \mathscr{G},
$$

such that the following properties are satisfied:

(1) $s\left(\gamma_{1} \circ \gamma_{2}\right)=s\left(\gamma_{2}\right)$ and $r\left(\gamma_{1} \circ \gamma_{2}\right)=r\left(\gamma_{1}\right)$, for any $\left(\gamma_{1}, \gamma_{2}\right) \in \varphi^{(2)}$;

(2) $s(x)=r(x)=x$ for any $x \in \varphi^{(0)}$;

(3) $\gamma \circ s(\gamma)=r(\gamma) \circ \gamma=\gamma$ for any $\gamma \in \mathscr{G}$;

(4) $\left(\gamma_{1} \circ \gamma_{2}\right) \circ \gamma_{3}=\gamma_{1} \circ\left(\gamma_{2} \circ \gamma_{3}\right)$;

(5) each element $\gamma \in \mathscr{G}$ has a two-sided inverse $\gamma^{-1}$ so that $\gamma \circ \gamma^{-1}=r(\gamma)$ and $\gamma^{-1} \circ \gamma=s(\gamma)$.

The groupoids interpolate between spaces and groups. This aspect especially pertains in the construction by Connes [1994, Section II.5] (see also [Hilsum and Skandalis 1987]) of the tangent groupoid $\mathscr{G}=\mathscr{G} M$ of a smooth manifold $M$.

At the set-theoretic level we let

$$
\varphi=T M \sqcup(M \times M \times(0, \infty)) \quad \text { and } \quad \varphi^{(0)}=M \times[0, \infty),
$$


where $T M$ denotes the (total space) of the tangent bundle of $M$. The inclusion $\iota$ of $\varphi^{(0)}$ into $\varphi$ is given by

$$
\iota(m, t)= \begin{cases}(m, m, t) & \text { for } t>0 \text { and } m \in M, \\ (m, 0) \in T M & \text { for } t=0 \text { and } m \in M .\end{cases}
$$

The range and source maps of $\mathscr{G}$ are such that

$$
\begin{aligned}
& r(p, q, t)=(p, t) \quad \text { and } \quad s(p, q, t)=(q, t) \quad \text { for } t>0 \text { and } p, q \in M, \\
& r(p, X)=s(p, X)=(p, 0) \quad \text { for } t=0 \text { and }(p, X) \in T M,
\end{aligned}
$$

while the composition law is given by

$$
\begin{aligned}
& (p, m, t) \circ(m, q, t)=(p, q, t) \quad \text { for } t>0 \text { and } m, p, q \in M, \\
& (p, X) \circ(p, Y)=(p, X+Y) \quad \text { for } t=0 \text { and }(p, X),(p, Y) \in T M .
\end{aligned}
$$

In fact, $\varphi M$ is a $b$-differentiable groupoid in the sense of the definition below.

Definition 3.2. A b-differentiable groupoid is a groupoid $\mathscr{G}$ so that $\mathscr{G}$ and $\mathscr{G}^{(0)}$ are smooth manifolds with boundary and the following properties hold:

(1) the inclusion of $\varphi^{(0)}$ into $\varphi$ is smooth;

(2) the source and range maps are smooth submersions, so that $\mathscr{G}^{(2)}$ is a submanifold (with boundary) of $\mathscr{G} \times \mathscr{G}$;

(3) the composition map $\circ: \mathscr{G}^{(2)} \rightarrow \mathscr{G}$ is smooth.

In the case of the tangent groupoid $\mathscr{G}=\mathscr{G} M$ the topology is such that:

- the inclusions of $\varphi^{(0)}$ and $\varphi^{(1)}:=M \times M \times(0, \infty)$ into $\varphi$ are continuous and $\mathscr{G}^{(1)}$ is an open subset of $\varphi$;

- a sequence $\left(p_{n}, q_{n}, t_{n}\right)$ from $\varphi^{(1)}$ converges to $(p, X) \in T M$ if, and only if, $\lim \left(p_{n}, q_{n}, t_{n}\right)=(p, p, 0)$ and for any local chart $\kappa$ near $p$ we have

$$
\lim _{n \rightarrow \infty} t_{n}^{-1}\left(\kappa\left(q_{n}\right)-\kappa\left(p_{n}\right)\right)=\kappa^{\prime}(p) X .
$$

One can check that this condition does not depend on the choice of a particular chart near $p$.

The differentiable structure of $\mathscr{G} M$ is obtained by gluing those of $T M$ and of $\varphi^{(1)}=M \times M \times(0, \infty)$ by means of a chart of the form,

$$
\gamma(p, X, t)= \begin{cases}\left(p, \exp _{p}(-t X), t\right) & \text { if } t>0 \text { and }(p, t X) \in \text { dom exp } \\ (p, X) & \text { if } t=0 \text { and }(p, X) \in \text { dom exp. }\end{cases}
$$

Here exp : dom exp $\rightarrow M \times M$ denotes the exponential map associated to an arbitrary Riemannian metric on $M$, so that $\gamma$ maps an open subset of $T M \times[0, \infty)$ 
onto an open neighborhood in $\mathscr{G}$ of the boundary $T M$ (see [Connes 1994], [Hilsum and Skandalis 1987], [Cariñena et al. 1999]).

3.2. The tangent groupoid of a Heisenberg manifold. We now construct the tangent groupoid $\varphi=\varphi_{H} M$ of a Heisenberg manifold $\left(M^{d+1}, H\right)$.

As an abstract groupoid $\mathscr{G}_{H} M$ is defined as follows. First, we set

$$
\varphi=G M \sqcup(M \times M \times(0, \infty)) \quad \text { and } \quad \varphi^{(0)}=M \times[0, \infty),
$$

where $G M$ denotes the (total space) of the tangent Lie group bundle of $M$. We have an inclusion $\iota: \mathscr{G}^{(0)} \rightarrow \mathscr{G}$ as in (3-1), namely,

$$
\iota(m, t)= \begin{cases}(m, m, t) & \text { for } t>0 \text { and } m \in M, \\ (m, 0) \in G M & \text { for } t=0 \text { and } m \in M .\end{cases}
$$

The range and source maps are defined similarly to (3-2)-(3-3) by letting

$$
\begin{aligned}
& r(p, q, t)=(p, t) \quad \text { and } \quad s(p, q, t)=(q, t) \quad \text { for } t>0 \text { and } p, q \in M, \\
& r(p, X)=s(p, X)=(p, 0) \quad \text { for } t=0 \text { and }(p, X) \in G M .
\end{aligned}
$$

In addition, we endow $\mathscr{G}$ with the composition law

$$
\begin{aligned}
& (p, m, t) \circ(m, q, t)=(p, q, t) \quad \text { for } t>0 \text { and } m, p, q \in M, \\
& (p, X) \circ(p, Y)=(p, X . Y) \quad \text { for } t=0 \text { and }(p, X),(p, Y) \in G M .
\end{aligned}
$$

It is immediate to check the properties (1)-(5) of Definition 3.1, noticing that the inverse map is here given by

$$
\begin{aligned}
& (p, q, t)^{-1}=(q, p, t) \quad \text { for } t>0 \text { and } p, q \in M, \\
& (p, X)^{-1}=\left(p, X^{-1}\right)=(p,-X) \quad \text { for } t=0 \text { and }(p, X) \in G M .
\end{aligned}
$$

Therefore, $\mathscr{G}=\mathscr{G}_{H} M$ is a groupoid.

Definition 3.3. The groupoid $\mathscr{G}_{H} M$ is called the tangent groupoid of $(M, H)$.

We now turn the groupoid $\mathscr{G}=\mathscr{G}_{H} M$ into a $b$-differentiable groupoid. First, we endow $\mathscr{G}$ with the topology such that:

- the inclusions of $\mathscr{G}^{(0)}$ and $\mathscr{G}^{(1)}:=M \times M \times(0, \infty)$ into $\mathscr{G}$ are continuous and make $\varphi^{(1)}$ an open subset of $\varphi$;

- a sequence $\left(p_{n}, q_{n}, t_{n}\right)$ from $\varphi^{(1)}$ converges to $(p, X) \in G M$ if, and only if, $\lim \left(p_{n}, q_{n}, t_{n}\right)=(p, p, 0)$ and for any local Heisenberg chart $\kappa: \operatorname{dom} \kappa \rightarrow U$ near $p$ we have

$$
\lim _{n \rightarrow \infty} t_{n}^{-1} \cdot \varepsilon_{\kappa\left(p_{n}\right)}\left(\kappa\left(q_{n}\right)\right)=\left(\varepsilon_{\kappa(p)} \circ \kappa\right)_{H}^{\prime}(p) X,
$$


where $t \cdot x$ is the Heisenberg dilation (2-17) and $\varepsilon_{u}$ denotes the coordinate change to the Heisenberg coordinates at $u \in U$ with respect to the $H$-frame of the Heisenberg chart $\kappa$ (see Definition 2.18).

Lemma 3.4. The condition (3-7) is independent of the choice of Heisenberg chart.

Proof. Assume that (3-7) holds for $\kappa$. Let $\kappa_{1}$ be another Heisenberg chart near $p$, and let $\phi=\kappa_{1} \circ \kappa^{-1}$. Letting $x_{n}=\kappa\left(p_{n}\right)$ and $y_{n}=\kappa\left(q_{n}\right)$, we have

$$
\begin{aligned}
t_{n}^{-1} \cdot \varepsilon_{\kappa_{1}\left(p_{n}\right)}\left(\kappa_{1}\left(q_{n}\right)\right) & =t_{n}^{-1} \cdot \varepsilon_{\phi\left(x_{n}\right)}\left(\phi\left(y_{n}\right)\right) \\
& =\delta_{t_{n}}^{-1} \circ \varepsilon_{\phi\left(x_{n}\right)} \circ \phi \circ \varepsilon_{x_{n}}^{-1} \circ \delta_{t_{n}}\left(t_{n} \cdot \varepsilon_{x_{n}}\left(y_{n}\right)\right) .
\end{aligned}
$$

On the other hand, since $\phi$ is a Heisenberg diffeomorphism it follows from Proposition 2.23 that as $t$ goes to zero we have

$$
\delta_{t}^{-1} \circ \varepsilon_{\phi(x)} \circ \phi \circ \varepsilon_{x}^{-1} \circ \delta_{t}(y)-\partial_{y}\left(\varepsilon_{\phi(x)} \circ \phi \circ \varepsilon_{x}^{-1}\right)_{H}(0) y \longrightarrow 0,
$$

locally uniformly with respect to $x$ and $y$. Since $\left(x_{n}, y_{n}, t_{n}\right) \rightarrow(\kappa(p), \kappa(p), 0)$ and $t_{n}^{-1} \cdot \varepsilon_{\kappa\left(p_{n}\right)}\left(\kappa\left(q_{n}\right)\right) \rightarrow\left(\varepsilon_{\kappa(p)} \circ \kappa\right)_{H}^{\prime}(p) X$, by combining this with (3-8) we get

$$
\begin{aligned}
\lim _{n \rightarrow \infty} t_{n}^{-1} \cdot \varepsilon_{\kappa_{1}\left(p_{n}\right)}\left(\kappa_{1}\left(q_{n}\right)\right) & =\left(\varepsilon_{\phi(\kappa(p))} \circ \phi \circ \varepsilon_{\kappa(p)}^{-1}\right)_{H}^{\prime}(0)\left(\left(\varepsilon_{\kappa(p)} \circ \kappa\right)_{H}^{\prime}(p) X\right) \\
& =\left(\varepsilon_{\kappa_{1}(p)} \circ \kappa_{1}\right)_{H}^{\prime}(p) X .
\end{aligned}
$$

Hence the lemma.

Next, in order to endow $\mathscr{G}_{H} M$ with a manifold structure we cannot make use of an exponential chart as in (3-4) because, unless $G M$ is a fiber bundle, the Liealgebraic structures of its fibers vary from point to point. Instead we can proceed as follows.

Let $\kappa: \operatorname{dom} \kappa \rightarrow U$ be a local Heisenberg chart near $m \in M$. We get a local coordinate system near $G M_{\mid \operatorname{dom} \kappa} \subset \mathscr{G}$ by letting

$$
\gamma_{\kappa}(x, X, t)= \begin{cases}\left(\kappa^{-1}(x), \kappa^{-1} \circ \varepsilon_{x}^{-1}(t \cdot X), t\right) & \text { if } t>0 \text { and } x, \varepsilon_{x}^{-1}(t \cdot X) \in U, \\ \left(\kappa^{-1}(x),\left(\kappa^{-1} \circ \varepsilon_{x}^{-1}\right)_{H}^{\prime}(0) X\right) & \text { if } t=0 \text { and }(x, X) \in U \times \mathbb{R}^{d+1}\end{cases}
$$

The map $\gamma_{\kappa}$ is one-to-one from an open neighborhood of the boundary $U \times \mathbb{R}^{d+1} \times 0$ in $U \times \mathbb{R}^{d+1} \times[0, \infty)$. Moreover, $\gamma_{\kappa}$ is continuous off the boundary. It is also continuous near any boundary point $(x, X, 0)$ because if a sequence $\left(x_{n}, X_{n}, t_{n}\right) \in$ dom $\gamma_{\kappa}$ with $t_{n}>0$ converges to $(x, X, 0)$ then $\left(p_{n}, q_{n}, t_{n}\right)=\gamma_{\kappa}\left(x_{n}, X_{n}, t_{n}\right)$ has limit $\left(\kappa^{-1}(x),\left(\kappa^{-1}\right)_{H}^{\prime}(x) X\right)=\gamma_{\kappa}(x, X, 0)$, for we have

$$
t_{n}^{-1} \cdot \varepsilon_{\kappa\left(p_{n}\right)}\left(\kappa\left(q_{n}\right)\right)=X_{n} \longrightarrow X=\kappa_{H}^{\prime}(\kappa(x))\left(\kappa^{-1}\right)_{H}^{\prime}(x) X .
$$


The inverse $\gamma_{\kappa}^{-1}$ is given by

$$
\begin{aligned}
& \gamma_{\kappa}^{-1}(p, q, t)=\left(\kappa(p), t^{-1} \cdot \varepsilon_{\kappa}(p) \circ \kappa(q), t\right) \text { for } t>0, \\
& \gamma_{\kappa_{1}}^{-1}(p, X)=\left(\kappa(p), \kappa_{H}^{\prime}(p) X\right) \text { for }(p, X) \in G M \text { in the range of } \gamma_{\kappa_{1}} .
\end{aligned}
$$

Therefore, if $\kappa_{1}$ is another local Heisenberg chart near $m$ then, in terms of $\phi=$ $\kappa_{1}^{-1} \circ \kappa$, the transition map $\gamma_{\kappa}^{-1} \circ \gamma_{\kappa_{1}}$ is

$$
\gamma_{\kappa}^{-1} \circ \gamma_{\kappa_{1}}(x, X, t)= \begin{cases}\left(\phi(x), t^{-1} \cdot \varepsilon_{\phi(x)} \circ \phi \circ \varepsilon_{x}^{-1}(t \cdot X), t\right) & \text { for } t>0, \\ \left(\phi(x), \phi_{H}^{\prime}(x) X, 0\right) & \text { for } t=0 .\end{cases}
$$

This shows that $\gamma_{\kappa}^{-1} \circ \gamma_{\kappa_{1}}(x, X, t)$ is smooth with respect to $x$ and $X$ and is meromorphic with respect to $t$ with at worst a possible singularity at $t=0$ only. However, by Proposition 2.23 we have

$$
\lim _{t \rightarrow 0} t^{-1} \cdot \varepsilon_{\phi(x)} \circ \phi \circ \varepsilon_{x}^{-1}(t \cdot X)=\phi_{H}^{\prime}(x) X,
$$

so there is no singularity at $t=0$. Hence $\gamma_{\kappa}^{-1} \circ \gamma_{\kappa_{1}}$ is a smooth diffeomorphism between open subsets of $\mathbb{R}^{d+1} \times[0, \infty)$. Therefore the coordinates system $\gamma_{\kappa}$ allows us to glue together the differentiable structures of $G M$ and $\varphi^{(1)}=M \times M \times(0, \infty)$ to turn $\mathscr{G}$ into a smooth manifold with boundary.

Next, $\varphi^{(0)}=M \times[0, \infty)$ is a manifold with boundary and the inclusion $\iota: \varphi^{(0)} \rightarrow \varphi$ is smooth. In addition, the range map $r$ and the source maps $s$ are submersions off the boundary. Moreover, in a coordinate system $\gamma_{\kappa}$ near the boundary of $\varphi$ the maps $r$ and $s$ are given by

$$
r(x, X, t)=(x, t) \quad \text { and } \quad s(x, X, t)=\left(\varepsilon_{x}^{-1}(t \cdot X), t\right),
$$

which shows that $\partial_{x, t} r$ and $\partial_{X, t} s$ are invertible near the boundary. Hence $r$ and $s$ are submersions on all $\varphi$.

Let us now look at the smoothness of the composition map.

Proposition 3.5. The composition map $\circ: \mathscr{G}^{2} \rightarrow \varphi_{\text {is smooth. }}$

Proof. Since $\circ$ is clearly smooth off the boundary we only need to understand what happens near the boundary. Using (3-11) we see that in a local coordinate system $\gamma_{\kappa}$ near the boundary two elements $(x, X, t)$ and $(y, Y, t)$ can be composed if, and only if, we have $y=\varepsilon_{x}(t \cdot X)$. Then for $t>0$ using (3-5) and (3-9) we see that $(x, X, t) \circ\left(\varepsilon_{x}^{-1}(t \cdot X), Y, t\right)$ is equal to

$$
\begin{aligned}
\gamma_{\kappa}^{-1}\left(( \kappa ^ { - 1 } ( x ) , \kappa ^ { - 1 } \varepsilon _ { x } ^ { - 1 } ( t \cdot X ) , t ) \circ \left(\kappa^{-1} \varepsilon_{x}^{-1}(\right.\right. & \left.\left.t \cdot X), \kappa^{-1} \circ \varepsilon_{\varepsilon_{x}^{-1}(t \cdot X)}^{-1}(t \cdot Y), t\right)\right) \\
& =\gamma_{\kappa}^{-1}\left(\left(\kappa^{-1}(x), \kappa^{-1} \circ \varepsilon_{\varepsilon_{x}^{-1}(t \cdot X)}^{-1}(t \cdot Y), t\right)\right) \\
& =\left(x, t^{-1} \cdot \varepsilon_{x} \circ \varepsilon_{\varepsilon_{x}^{-1}(t \cdot X)}^{-1}(t \cdot Y), t\right) .
\end{aligned}
$$


On the other hand, for $t=0$ from (3-6) and (3-10) we see that $(x, X, 0) \circ(x, Y, 0)$ is equal to

$$
\begin{aligned}
\gamma_{\kappa}^{-1}\left(\left(\kappa^{-1},\left(\kappa^{-1} \circ \varepsilon_{x}^{-1}\right)_{H}^{\prime}(0) X\right) \circ\left(\kappa^{-1},\left(\kappa^{-1} \circ \varepsilon_{x}^{-1}\right)_{H}^{\prime}(0) Y\right)\right) \\
=\gamma_{\kappa}^{-1}\left(\left(\kappa^{-1}(x),\left(\left(\kappa^{-1} \circ \varepsilon_{x}^{-1}\right)_{H}^{\prime}(0) X\right) \cdot\left(\left(\kappa^{-1} \circ \varepsilon_{x}^{-1}\right)_{H}^{\prime}(0) Y\right)\right)\right. \\
=\gamma_{\kappa}^{-1}\left(\kappa^{-1}(x),\left(\kappa^{-1} \circ \varepsilon_{x}^{-1}\right)_{H}^{\prime}(0)(X \cdot Y)\right) \\
=(x, X \cdot Y, 0),
\end{aligned}
$$

where we have used the fact that $\left(\kappa^{-1} \circ \varepsilon_{x}^{-1}\right)_{H}^{\prime}(0)$ is a morphism of Lie groups (cf. Proposition 2.12). Therefore, we get

$$
(x, X, t) \circ\left(\varepsilon_{x}^{-1}(t \cdot X), Y, t\right)= \begin{cases}\left(x, t^{-1} \cdot \varepsilon_{x} \circ \varepsilon_{\varepsilon_{x}^{-1}(t \cdot X)}^{-1}(t \cdot Y), t\right) & \text { for } t>0, \\ (x, X \cdot Y, 0) & \text { for } t=0 .\end{cases}
$$

This shows that $\circ$ is smooth with respect to $x, X$, and $Y$ and is meromorphic with respect to $t$ with at worst a singularity at $t=0$. Therefore, in order to show the smoothness of $\circ$ at $t=0$ it is enough to prove that

$$
\lim _{t \rightarrow 0^{+}} t^{-1} \cdot \varepsilon_{x} \circ \varepsilon_{\varepsilon_{x}^{-1}(t \cdot X)}^{-1}(t \cdot Y)=X \cdot Y .
$$

Lemma 3.6. Let $\psi_{u}$ denote the affine change to the privileged coordinates at $u$ as in Definition 2.15. Then with respect to the law group of the u-group $G^{(u)}$ we have

$$
\lim _{t \rightarrow 0} t^{-1} \cdot \psi_{u} \circ \psi_{\psi_{u}^{-1}(t . v)}^{-1}(t . w)=v . w,
$$

locally uniformly with respect to $w$.

Proof. Let $\lambda_{v}(w)=v \cdot w$ and $\mu_{t}(w)=t^{-1} \cdot \psi_{u} \circ \psi_{\psi_{u}^{-1}(t \cdot v)}^{-1}(t \cdot w)$. For $w=0$ we have

$$
\mu_{t}(0)=t^{-1} \cdot \psi_{u} \circ \psi_{\psi_{u}^{-1}(t \cdot v)}^{-1}(0)=t^{-1} \cdot \psi_{u}\left(\psi_{u}^{-1}(t \cdot v)\right)=v=\lambda_{v}(0) .
$$

Remark also that $\mu_{t}$ and $\lambda_{v}$ are both affine maps and we have

$$
\mu_{t}^{\prime}=\delta_{t}^{-1} \circ \psi_{u}^{\prime} \circ\left(\psi_{\psi_{u}^{-1}(t \cdot v)}^{-1}\right)^{\prime} \circ \delta_{t} .
$$

Let $X_{0}, \ldots, X_{d}$ be the $H$-frame associated to the Heisenberg chart $\kappa$ (seen as an $H$-frame on $U=$ range $\kappa)$ and set $w_{0}=2$ and $w_{1}=\cdots=w_{d}=1$. By (2-18) and (2-19) we have $X_{j}(u)=\left(\psi_{u}^{-1}\right)^{\prime}\left(\partial_{x_{j}}\right)$ for $j=0, \ldots, d$. Therefore, we get

$$
\left(\delta_{t}^{*} \psi_{u *} X_{j}\right)(v)=\delta_{t}^{-1} \circ \psi_{u}^{\prime}\left(X_{j}\left(\psi_{u}^{-1} \circ \delta_{t}(v)\right)\right)=\delta_{t}^{-1} \circ \psi_{u}^{\prime} \circ\left(\psi_{\psi_{u}^{-1}(t \cdot v)}^{-1}\right)^{\prime}\left(\partial_{x_{j}}\right) .
$$


Combining with (3-15) we thus obtain

$$
\begin{aligned}
t^{w_{j}}\left(\delta_{t}^{*} \psi_{u *} X_{j}\right)(v) & =\delta_{t}^{-1} \circ \psi_{u}^{\prime} \circ\left(\psi_{\psi_{u}^{-1}(t \cdot v)}^{-1}\right)^{\prime}\left(t^{w_{j}} \partial_{x_{j}}\right) \\
& =\delta_{t}^{-1} \circ \psi_{u}^{\prime} \circ\left(\psi_{\psi_{u}^{-1}(t \cdot v)}^{-1}\right)^{\prime} \circ \delta_{t}\left(\partial_{x_{j}}\right)=\mu_{t}^{\prime}\left(\partial_{x_{j}}\right) .
\end{aligned}
$$

Now, for $j=1, \ldots, d$, let $X_{j}^{(u)}$ be the left-invariant field on $G^{(u)}$ with $X_{j}^{(u)}=\partial_{x_{j}}$. Recall that, by the very definition of $G^{(u)}$ we have $X_{j}^{(u)}=\lim _{t \rightarrow 0} t^{w_{j}}\left(\delta_{t}^{*} \psi_{u *} X_{j}\right)$. Thus,

$$
X_{j}^{(u)}(v)=\lim _{t \rightarrow 0} \mu_{t}^{\prime}\left(\partial_{x_{j}}\right)
$$

In fact, as $X_{j}^{(u)}$ is left-invariant, we have

$$
X_{j}^{(u)}(v)=\left(\lambda_{v *} X_{j}^{(u)}\right)(v)=\lambda_{v}^{\prime}\left(X_{j}^{(u)}(0)\right)=\lambda_{v}^{\prime}\left(\partial_{x_{j}}\right) .
$$

Therefore, we have $\lim _{t \rightarrow 0} \mu_{t}^{\prime}\left(\partial_{x_{j}}\right)=\lambda_{v}^{\prime}\left(\partial_{x_{j}}\right)$ for $j=0, \ldots, d$, which yields

$$
\lim _{t \rightarrow 0} \mu_{t}^{\prime}=\lambda_{v}^{\prime} .
$$

Since by (3-14) we have $\mu_{t}(0)=\lambda_{v}(0)$ and since $\mu_{t}$ and $\lambda_{v}$ are affine maps, it follows that as $t$ goes to zero $\mu_{t}(w)=t^{-1} \cdot \psi_{u} \circ \psi_{\psi_{u}^{-1}(t \cdot v)}^{-1}(t \cdot w)$ converges to $\lambda_{v}(w)=$ $v \cdot w$ locally uniformly with respect to $w$. Hence the lemma.

Next, let $\phi_{x}$ be the map (2-27), that is, the transition map from $x$-coordinates to Heisenberg coordinates centered at $x$. Recall that $\phi_{x}$ is an isomorphism of graded Lie groups from $G^{(x)}$ to the tangent group $G_{x}=\left(\kappa_{*} G M\right)_{x}$. Therefore, as $\varepsilon_{x}=$ $\phi_{x} \circ \psi_{x}$ we get

$$
\begin{aligned}
t^{-1} \cdot \varepsilon_{x} \circ \varepsilon_{\varepsilon_{x}^{-1}(t \cdot X)}^{-1}(t \cdot Y) & =\delta_{t}^{-1} \circ \phi_{x} \circ \psi_{x} \circ \psi_{\psi_{x}^{-1} \circ \phi_{x}(t \cdot X)}^{-1} \circ \phi_{\varepsilon_{x}^{-1}(t \cdot X)} \circ \delta_{t}(Y) \\
& =\phi_{x}\left(\delta_{t}^{-1} \circ \psi_{x} \circ \psi_{\psi_{x}^{-1}(t \cdot v)}^{-1} \circ \delta_{t}\left(w_{t}\right)\right),
\end{aligned}
$$

where we have let $v=\phi_{x}^{-1}(X)$ and $w_{t}=\phi_{\varepsilon_{x}^{-1}(t \cdot X)}(Y)$. Combining this with (3-13) we get

$$
\lim _{t \rightarrow 0} t^{-1} \cdot \varepsilon_{x} \circ \varepsilon_{\varepsilon_{x}^{-1}(t \cdot X)}^{-1}(t \cdot Y)=\phi_{x}\left(v \cdot \lim _{t \rightarrow 0} w_{t}\right)=\phi_{x}\left(\phi_{x}^{-1}(X) \cdot \phi_{x}^{-1}(Y)\right)=X \cdot Y .
$$

This proves (3-12) and thus completes the proof of Proposition 3.5.

Summarizing all this we have proved:

Theorem 3.7. The groupoid $\mathscr{G}_{H} M$ is a b-differentiable groupoid.

Finally, let $\phi$ be a Heisenberg diffeomorphism from $(M, H)$ onto a Heisenberg manifold $\left(M^{\prime}, H^{\prime}\right)$ and let us compare the tangent groupoids $\varphi_{H} M$ and $\varphi_{H^{\prime}} M^{\prime}$. To 
this end consider the map $\Phi_{H}: \mathscr{G}_{H} M \rightarrow \mathscr{G}_{H^{\prime}} M^{\prime}$ given by

$$
\begin{array}{ll}
\Phi_{H}(p, q, t)=(\phi(p), \phi(q), t) & \text { for } t>0 \text { and } p, q \in M, \\
\Phi_{H}(p, X)=\left(\phi(p), \phi_{H}^{\prime}(p) X\right) & \text { for }(p, X) \in G M .
\end{array}
$$

For $t>0$ and $p, q \in M$ we have

$$
\begin{aligned}
& r_{M^{\prime}} \circ \Phi_{H}(p, q, t)=(\phi(q), t)=\Phi_{H} \circ r_{M}(p, q, t), \\
& s_{M^{\prime}} \circ \Phi_{H}(p, q, t)=(\phi(p), t)=\Phi_{H} \circ s_{M}(p, q, t),
\end{aligned}
$$

while for $(p, X) \in G M$ we have

$$
\begin{aligned}
s_{M^{\prime}} \circ \Phi_{H}(p, X) & =r_{M^{\prime}} \circ \Phi_{H}(p, X)=(\phi(p), 0) \\
& =\Phi_{H} \circ r_{M}(p, X)=\Phi_{H} \circ s_{M}(p, X) .
\end{aligned}
$$

Hence $r_{M^{\prime}} \circ \Phi_{H}=\Phi_{H} \circ r_{M}$ and $s_{M^{\prime}} \circ \Phi_{H}=\Phi_{H^{\circ}} \circ s_{M}$. Incidentally $\Phi_{H}\left(\varphi_{H}^{(2)} M\right)$ agrees with $\varphi_{H^{\prime}}^{(2)} M^{\prime}$.

Moreover, for $t>0$ and $m, p, q \in M$ we have

$$
\begin{aligned}
\Phi_{H}(m, p, t) \circ_{M^{\prime}} \Phi_{H}(p, q, t) & =(\phi(m), \phi(q), t) \\
& =\Phi_{H}\left((m, p, t) \circ_{M}(p, q, t)\right),
\end{aligned}
$$

and for $p \in M$ and $X, Y \in G_{p} M$ we get

$$
\begin{aligned}
\Phi_{H}(p, X) \circ_{M^{\prime}} \Phi_{H}(p, Y) & =\left(\phi(p), \phi_{H}^{\prime}(p)(X \cdot Y)\right) \\
& =\Phi_{H}\left((p, X) \circ_{M} \Phi_{H}(p, Y)\right) .
\end{aligned}
$$

All this shows that $\Phi_{H}$ is a morphism of groupoids. In fact, the map defined by replacing $\phi$ with $\phi^{-1}$ in (3-16) and (3-17) is an inverse for $\Phi_{H}$, so $\Phi_{H}$ is in fact a groupoid isomorphism from $\varphi_{H} M$ onto $\varphi_{H^{\prime}} M^{\prime}$.

Next, it follows from (3-16) that $\Phi_{H}$ is continuous off the boundary. To see what happens at the boundary consider a sequence $\left(p_{n}, q_{n}, t_{n}\right)$ converging to $(p, X) \in$ $G M$ and let $\kappa$ be a local Heisenberg chart for $M^{\prime}$ near $p^{\prime}=\phi(p)$. By pulling back the $H^{\prime}$-frame of $\kappa$ by $\phi$ we turn $\kappa \circ \phi$ into a Heisenberg chart so setting $\left(p_{n}^{\prime}, q_{n}^{\prime}, t_{n}\right)=\Phi_{H}\left(p_{n}, q_{n}, t_{n}\right)$ we get

$t_{n}^{-1} \cdot \varepsilon_{\kappa\left(p_{n}^{\prime}\right)}\left(\kappa\left(q_{n}^{\prime}\right)\right)=t_{n} \cdot \varepsilon_{\kappa \circ \phi\left(p_{n}\right)}\left(\kappa \circ \phi\left(q_{n}\right)\right) \longrightarrow(\kappa \circ \phi)_{H}^{\prime}(p) X=\kappa_{H}^{\prime}(p)\left(\phi_{H}^{\prime}(p) X\right)$.

Thus, $\Phi_{H}$ is continuous from $\mathscr{G}_{H} M$ to $\mathscr{G}_{H^{\prime}} M^{\prime}$.

It also follows from (3-16) that $\Phi_{H}$ is smooth off the boundary. Moreover, if $\kappa$ is a local Heisenberg chart for $M^{\prime}$ then $\Phi_{H} \circ \gamma_{\kappa \circ \phi}(p, X, t)$ coincides for $t>0$ with

$$
\begin{aligned}
\left(\phi\left(\phi^{-1} \circ \kappa^{-1}(x)\right), \phi\left(\phi^{-1} \circ \kappa^{-1} \circ \varepsilon_{x}^{-1}(t \cdot X)\right), t\right) & =\left(\kappa^{-1}(x), \kappa^{-1} \circ \varepsilon_{x}^{-1}(t \cdot X), t\right) \\
& =\gamma_{\kappa}(x, X, t),
\end{aligned}
$$


while for $t=0$ it is equal to

$$
\begin{aligned}
\left(\phi\left(\phi^{-1} \circ \kappa^{-1}(x)\right), \phi_{H}^{\prime}\left(\phi^{-1} \circ \kappa^{-1}(x)\right)\right. & \left.\left(\left(\kappa^{-1} \circ \varepsilon_{x}^{-1}\right)_{H}^{\prime}(0) X\right), 0\right) \\
= & \left(\kappa^{-1}(x),\left(\kappa^{-1} \circ \varepsilon_{x}^{-1}\right)_{H}^{\prime}(0) X, t\right)=\gamma_{\kappa}(x, X, 0) .
\end{aligned}
$$

Hence $\gamma_{\kappa} \circ \Phi \circ \gamma_{\kappa \circ \phi}=\mathrm{id}$, which shows that $\Phi_{H}$ is smooth map. Since similar arguments show that $\Phi_{H}^{-1}$ is smooth, it follows that $\Phi_{H}$ is a diffeomorphism. We have thus proved:

Proposition 3.8. The map $\Phi_{H}: \mathscr{G}_{H} M \rightarrow \mathscr{G}_{H^{\prime}} M^{\prime}$ given by (3-16)-(3-17) is an isomorphism of b-differentiable groupoids. Hence the isomorphism class of b-groupoids of $\varphi_{H} M$ depends only on the Heisenberg diffeomorphism class of $(M, H)$.

\section{References}

[Atiyah and Singer 1968a] M. F. Atiyah and I. M. Singer, "The index of elliptic operators, I", Ann. of Math. (2) 87 (1968), 484-530. MR 38 \#5243 Zbl 0164.24001

[Atiyah and Singer 1968b] M. F. Atiyah and I. M. Singer, "The index of elliptic operators, III", Ann. of Math. (2) 87 (1968), 546-604. MR 38 \#5245 Zbl 0164.24301

[Beals and Greiner 1988] R. Beals and P. Greiner, Calculus on Heisenberg manifolds, Ann. of Math. Studies 119, Princeton University Press, Princeton, NJ, 1988. MR 89m:35223 Zbl 0654.58033

[Bellaïche 1996] A. Bellaïche, "The tangent space in sub-Riemannian geometry", pp. 1-78 in SubRiemannian geometry, edited by A. Bellaïche and J.-J. Risler, Progr. Math. 144, Birkhäuser, Basel, 1996. MR 98a:53108 Zbl 0862.53031

[Cariñena et al. 1999] J. F. Cariñena, J. Clemente-Gallardo, E. Follana, J. M. Gracia-Bondía, A. Rivero, and J. C. Várilly, "Connes' tangent groupoid and strict quantization”, J. Geom. Phys. 32:2 (1999), 79-96. MR 2000i:53127 Zbl 0961.53047

[Connes 1994] A. Connes, Noncommutative geometry, Academic Press, San Diego, CA, 1994. MR 95j:46063 Zbl 0818.46076

[Eliashberg and Thurston 1998] Y. M. Eliashberg and W. P. Thurston, Confoliations, University Lecture Series 13, American Math. Society, Providence, RI, 1998. MR 98m:53042 Zbl 0893.53001

[Epstein et al. 1991] C. L. Epstein, R. B. Melrose, and G. A. Mendoza, "Resolvent of the Laplacian on strictly pseudoconvex domains", Acta Math. 167 (1991), 1-106. MR 92i:32016 Zbl 0758.32010

[Folland and Stein 1974] G. B. Folland and E. M. Stein, "Estimates for the $\bar{\partial}_{b}$ complex and analysis on the Heisenberg group", Comm. Pure Appl. Math. 27 (1974), 429-522. MR 51 \#3719 Zbl 0293.35012

[Gromov 1996] M. Gromov, "Carnot-Carathéodory spaces seen from within”, pp. 79-323 in SubRiemannian geometry, edited by A. Bellaïche and J.-J. Risler, Progr. Math. 144, Birkhäuser, Basel, 1996. MR 2000f:53034 Zbl 0864.53025

[Hilsum and Skandalis 1987] M. Hilsum and G. Skandalis, "Morphismes $K$-orientés d'espaces de feuilles et fonctorialité en théorie de Kasparov (d'après une conjecture d'A. Connes)", Ann. Sci. École Norm. Sup. (4) 20:3 (1987), 325-390. MR 90a:58169 Zbl 0656.57015

[Ponge 2000] R. Ponge, Calcul hypoelliptique sur les variétés de Heisenberg, résidu non commutatif et géométrie pseudo-hermitienne, Ph.D. thesis, University of Paris-Sud (Orsay), 2000. 
[Rockland 1987] C. Rockland, "Intrinsic nilpotent approximation", Acta Appl. Math. 8:3 (1987), 213-270. MR 88i:35032 Zbl 0621.22010

[Taylor 1984] M. E. Taylor, "Noncommutative microlocal analysis, I", Mem. Amer. Math. Soc. 52:313 (1984), iv+182. MR 86f:58156 Zbl 0554.35025

[Van Erp 2005] E. Van Erp, The Atiyah-Singer index formula for subelliptic operators on contact manifolds, Ph.D. thesis, Pennsylvania State University, 2005.

Received August 30, 2004. Revised May 9, 2005.

RAPHAËL PONGE

DEPARTMENT OF MATHEMATICS

OHIO STATE UNIVERSITY

Columbus, OH 43210-1174

UNITED STATES

ponge@math.osu.edu 